\title{
Die Kommunalisierung und ihre sozialwirtschaftliche Antwort
}

VON HEINZ-JÜRGEN DAHME UND NORBERT WOHLFAHRT

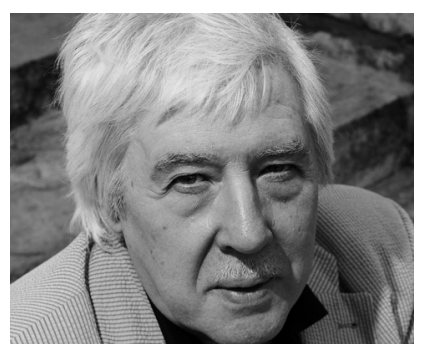

Prof. Dr. Dahme lehrt an der Hochschule Magdeburg-Stendal mit den Schwerpunkten Verwaltungswissenschaft, Wohlfahrtsverbände, Verwaltungsmanagement und Sozialplanung. E-Mail heinz-juergen.dahme@ hs-magdeburg.de

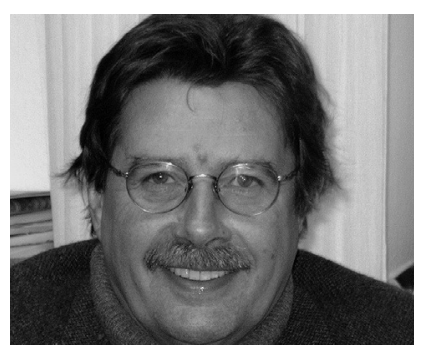

Prof. Dr. Norbert Wohlfahrt ist Hochschullehrer für Sozialmanagement an der Evangelischen Fachhochschule Rheinland-Westfalen-Lippe. Seine Forschungsschwerpunkte sind kommunale Sozialpolitik und Sozialverwaltung sowie die Entwicklung sozialer Dienste und von Non-Profit-Organisationen.

E-Mail

wohlfahrt@efh-bochum.de
Die Kommunalisierungspolitik hat massive Wirkungen auf die Träger sozialer Dienste und Einrichtungen. Der Aufbau eines eigenen Lobbying im Sinne der Anwaltsfunktion für benachteiligte Menschen wird zu einem Bestandteil der Portfolio-Struktur jedes einzelnen Sozialunternehmen.

Die Ausgaben der Gebietskörperschaften im Bereich sozialer Leistungen werden auch in Zukunft ungebrochen wachsen, so die Prognosen der Kommunalen Spitzenverbände. Angesichts der zu erwartenden Mindereinnahmen aufgrund von Steuerausfällen wie durch die abnehmende Wirtschaftsleistung werden die kommunalen Sozialausgaben weiter unter Druck geraten. Die hohe Staatsverschuldung aufgrund der Finanzmarktkrise wird eine Debatte über soziale Leistungen, ihre Höhe wie ihren Umfang hervorrufen, in der man zu drastischen Schlussfolgerungen kommen wird. Perspektivisch ist davon auszugehen, dass zukünftig nur noch Pflichtaufgaben finanziert werden und dass das Lobbying der Verbände vor neuen Herausforderungen steht.

\section{These 1:}

\section{Die Kommunalisierungspolitik verstärkt das Aufkommen neuer sozialpolitischer Leitbilder}

Die Sparhaushalte auf Landes- und Kommunalebene zu Beginn der 1990er Jahre haben die Debatte über die Kommunalisierung von Leistungen und Förderstrukturen aufkommen lassen. Folge dieser Finanzsituation war, dass die Kommunalverwaltungen bereit waren, neue Steuerungs- und Managementmodelle (Neues Steuerungsmodell, New Public Management) zu implementieren, um ihr Handeln wirtschaftlicher und effizienter zu gestalten.

Hinzu kam die Überzeugung, dass sich soziale Dienste besser und wirkungsvoller ortsnah steuern ließen als über landesweite Programme und Standards. Lokale Armutsberichte zeigten ortsnahe Bedarfe auf, die durch Bürgerengagement und eine opti- mierte Vernetzung lokaler Leistungserbringer wirksamer und kostengünstiger bearbeitet werden sollten.

Auf der kommunalen Ebene gab es bereits seit Mitte der 1990er Jahre die Einführung von Festbeträgen bei der Finanzierung freier Träger; auch wurden Standards zurückgenommen, um Trägern Spielraum für kostengünstigeres und flexibleres Arbeiten zu ermöglichen. Die Kritik an stetig wachsenden zentralen Steuerungsverfahren der Länder nahm zu, da die Steuerung der Kommunen durch die Länder den Widerspruch zum grundgesetzlich verankerten Konnexitätsprinzip (1) deutlich werden ließ.

Für die Entwicklung der Kommunalisierungspolitik - also die zunehmende Dezentralisierung von sozialen Aufgaben und Verantwortungsdelegation an die lokale Ebene - war aber vor allem entscheidend, dass das im Grundgesetz verankerte Gebot der »Einheitlichkeit der Lebensverhältnisse «, das unmittelbare Auswirkungen auf das Sozialstaatsgebot hat, gelockert und ersetzt wurde durch das Gebot der Herstellung "gleichwertiger Lebensverhältnisse « (Art. 72 Abs. 2 GG). Der ehemals kooperative Föderalismus verändert sich damit hin zu einem konkurrenziellen Föderalismus. Laut Bundesverfassungsgericht sind partikular-differenzierte Lebensverhältnisse in der Bundesrepublik nunmehr ausdrücklich erwünscht.

Aktivierende Sozialpolitik, Bürgergesellschaft und Bürgerkommune, Kommunalisierung von Aufgaben, all diese Konzepte und Prozesse konfrontieren die Kommunalverwaltung nicht nur mit ökonomisch-betriebswirtschaftlichen Fragen, sondern fordern sie heraus, neue sozial-, gesellschafts- wie gerechtigkeitspolitische Antworten auf die durch globale Entwicklungen verursachten lokalen Veränderungen und Transformationsprozesse zu entwickeln, da der nationale Sozialstaat sich selbst durch die Verantwortungsverlagerung nach unten zunehmend entlastet.

Diese Entwicklung bringt neue Herausforderungen an die politische Steuerung in den Kommunen mit sich. Eines zeichnet sich deshalb schon deutlich ab: In dem Maße, wie sich ein parteiübergreifender 
Konsens zum Um- und Abbau des Sozialstaates auf Bundesebene entwickelt, wird es notwendig, auf kommunaler Ebene - in der Lebenswelt des Bürgers - eigene, lokale sozial-, gesellschafts- und gerechtigkeitspolitische Leitbilder und Interventionsprogramme $\mathrm{zu}$ entwickeln und $\mathrm{zu}$ implementieren, was sich beispielsweise an der aktuellen Konjunktur sogenannter kommunaler Integrationskonzepte, Arbeitsmarktpolitiken wie Sicherheits- und Ordnungsprogramme ablesen lässt.

\section{These 2:}

\section{Der Sozialstaatsumbau macht ein Change-Management in sozialen Diensten erforderlich}

Die Träger und Einrichtungen der Freien Wohlfahrtspflege haben in den vergangenen Jahren einen intensiven, staatlich induzierten Modernisierungsprozess durchlaufen. Hierbei ging es primär darum, aus weltanschaulich und sozialpolitisch begründeten gemeinnützigen Organisationen sozialwirtschaftliche Leistungserbringer zu formen, deren zentrale Aufgabe die Erbringung professioneller Dienstleistungen ist, die unter Effektivitäts- und Effizienzkriterien darstellbar und kontrollierbar sind.

Die neue wettbewerbliche Ordnungsstruktur hat Auswirkungen auf die Organisation sozialer Dienste und führt zur Etablierung eines Change-Managements: Neue wettbewerbsorientierte lokale Finanzierungsregeln, ein umfängliches Berichtswesen, die Einführung von Qualitätsmanagementsystemen in den Einrichtungen, ein verschärfter Kostenwettbewerb unter den Leistungserbringern und als Folge davon neue Entlohnungsregeln für die Mitarbeiter und Mitarbeiterinnen der Leistungserbringer u. Ä. haben sich im sozialen Dienstleistungssektor mittlerweile flächendeckend etabliert. Die Veränderungen wirken sich nicht nur auf das Verhältnis zwischen Kostenträgern und Leistungserbringern aus, sondern haben auch gravierende Auswirkungen auf die Organisation der Arbeit bei den Leistungserbringern selbst, deren Organisationsformen und Strukturen der Arbeitserledigung sich - trotz angeblicher Werteorientierung - immer ähnlicher werden.

"Structure follows function ", so könnte man die durch die neue Sozialpolitik und Steuerungsphilosophie ausgelöste Nivellierung der Leistungserbringung und Leistungsorganisation beschreiben. Leistungskontrollen, wirkungsorientierte Entgelte, Evaluation und Controlling im Rahmen des kommunalen strategischen Managements stärken merklich die öffentlichen Träger und verstärken den vom Kontraktmanagement eingeleiteten Prozess, das Verhältnis von öffentlichen und privaten Trägern durch die Etablierung eines Monitoring des Leistungsgeschehens zu transformieren.

Nachdem man innerhalb der Verbände jahrelang diskutiert hat, wie man sich zum wettbewerblich organisierten Sozialsystem positionieren soll, haben sich nun alle Verbände dazu durchgerungen, dieses als notwendige und positive Bedingung ihrer Arbeit anzuerkennen. Damit sind aber weitere Modernisierungsprozesse verbunden, die sich gegenwärtig auf verschiedenen Ebenen gleichzeitig vollziehen: die Implementation wichtiger betriebswirtschaftlicher Instrumentarien (Controlling, Qualitätsmanagement, Doppik) noch defizitär ist und eine Reihe von wichtigen Instrumenten zur Stärkung der Wettbewerbsfähigkeit noch wenig genutzt wird (z. B. strategische Steuerung, Risikomanagement, Personalentwicklung, Marketing).

Für den Gewährleistungsstaat und die neue Gewährleistungsverwaltung sind Träger und Einrichtungen Sozialunternehmen, die als Dienstleister staatliche Aufträge durchführen. Dieses neue Staatsverständnis
- Auf der Mikroebene führt der Professionalisierungsschub der Unternehmen zu einer immer stärkeren Ablösung von der wohlfahrtsverbandlichen Einbettung. Betriebliche Zielgrößen bestimmen zunehmend das Handeln. Zugleich verschärfen sich die verbandsinternen Konkurrenzen auf der lokalen oder regionalen Ebene.

- Auf der Meso-Ebene geht es um ein modernisiertes Konzept multifunktional bestimmter Verbandlichkeit. Sozialanwaltschaft und Dienstleistungsorientierung müssen koordiniert werden, während sich gleichzeitig die Interessen zwischen Fachverbänden und Dachverbänden auseinanderentwickeln. Das Konzept der kleinteilig versäulten Fachverbände scheint überholt und es bilden sich sektorale Verbandsstrukturen. Auch die Neugestaltung des Verhältnisses von Kirche und Verband wird hierdurch reformbedürftig.

- Auf der Makro-Ebene stellt sich die Frage nach der strategischen Positionierung der Freien Wohlfahrtspflege und sozialpolitisch wirksamen spitzenverbandlichen Strukturen.

\section{These 3:}

\section{Aktivierungspolitik und die} sozialpolitische Verantwortungsdelegation nach unten erfordert eine Neufassung der Anwaltsfunktion und neue Lobbyingstrukturen

Die Wohlfahrtsverbände sind inzwischen in der Sozialwirtschaft angekommen. Dennoch ist dieser Prozess noch nicht abgeschlossen, da die Wettbewerbsorientierung noch nicht flächendeckend nachweisbar ist, beinhaltet eine sozialpolitische Entwertung der Verbände, die sich von einigen zu Zeiten des Korporatismus entwickelten Aufgaben verabschieden oder diese neu definieren müssen, insbesondere die ihnen wichtige und ihr Selbstbild bestimmende Anwaltsfunktion. Die Anwaltsfunktion war im Selbstbild der Spitzenverbände der Freien Wohlfahrtspflege eng mit ihrer Dienstleistungsfunktion verknüpft. Sozialanwaltschaft und Leistungserbringung bilden eine Einheit, da Dienstleistung (Helfen) - vor allem im kirchlichen Kontext - Parteinahme für Klienten und Hilfebedürftige ist. (2)

Im aktivierenden Sozialstaat greift dieses Anwaltschaftsmodell nicht mehr, da sich Anwaltschaft nicht durch stellvertretendes Handeln kennzeichnen soll, sondern durch Befähigung (empowerment) und Unterstützung der Klienten, ihre Interessen selbst zu artikulieren und politisch zu vertreten. Im kommunalisierten Sozialstaat, der sich durch interessensorientierte strategische Allianzen und weniger durch verfestigte und werteorientierte korporatistische Strukturen kennzeichnen soll, ist die Anwaltsfunktion flexibilisiert und situativ definiert, kann nicht in korporatistische Strukturen eingebettet sein. Der dezentrale Sozialstaat - als Produkt der Kommunalisierung - macht die Entwicklung neuer Instrumente der politischen Einflussnahme notwenig und erfordert Kompetenzen strategischen Handelns, vor allem die Kompetenz, strategische Allianzen - auch mit lokalen Akteuren, wie Unternehmen und Gewerkschaften, die bislang nicht zu den Lieblingspartnern einiger Verbände gehörten - bilden zu können.

Die neue dezentrale Sozialstaatlichkeit vor Ort macht es erforderlich, das Lobbying der Verbände und Einrichtungen diffe- 
renziert zu organisieren und eine Funktionsdifferenzierung zwischen dem Lobbying für die Träger und Einrichtungen und dem Lobbying im Rahmen der Wahrnehmung sozialanwaltschaftlicher Aufgaben zu entwickeln. Beide Funktionen müssen im aktivierenden und lokal ausgerichteten Sozialstaatsmodell aus organisationspolitischer Sicht strikt unterschieden werden, weil sie in ihrem Vollzug keineswegs identisch sein müssen (was in der Vergangenheit häufig unterstellt wurde). Im Zuge einer weiter zunehmenden sozialwirtschaftlichen Entwicklung könnten beide Funktionen miteinander in Konflikt geraten, weil Klienteninteressen und betriebswirtschaftliche Interessen der Verbände und Einrichtungen nicht deckungsgleich sein müssen.

Voraussetzung eines effektiven Lobbying für Träger und Einrichtungen ist vor allem die verbandsinterne Trennung zwischen der das Lobbying betreibenden Organisationseinheit einerseits und der Betriebsebene andererseits. Die organisatorische Verselbständigung des Lobbying muss - aus Sicht einiger Verbandsstrategen - einhergehen mit einer systematischen Erfassung und Repräsentation der Trägerinteressen in den Strukturen der Spitzenverbände. Instrumente hierfür können Arbeitsgemeinschaften sein, die sich entlang der Trägerstrukturen in den verschiedenen Arbeitsfeldern sozialer Dienste konstituieren und deren Geschäftsführung durch den Spitzenverband wahrgenommen wird.

Die systematische Einbindung der Betriebsinteressen in die Arbeit des Verbandes (bei sozialen Großbetrieben ist es dementsprechend die Repräsentanz der operativen Ebene im strategischen Management) und die Meinungsbildung auf Spitzenverbandsebene bzw. Strategieebene ist Ausdruck des Tatbestandes, dass die Träger auf örtli- cher Ebene (Ortsverbände) stärker an den Verband heranrücken müssen und den Spitzenverband und seine örtlichen Untergliederungen stärker als bislang als Einheit begreifen müssen.

\section{These 4: \\ Die Kommunalisierungspolitik führt zur Wahrnehmung strategischer Aufgaben auf lokaler Ebene und macht die Integration gesellschaftspolitischer Aufgaben in die Sozialunternehmen notwenig}

Die durch die Kommunalisierungspolitik bewirkte Stärkung der lokalen Ebene in sozialpolitischer Hinsicht, bei gleichzeitigem Rückgang des Steueraufkommens und sich verschärfenden sozialen Problemlagen, bringt eine grundlegende veränderte Dynamik in die klassischen Aufbau- und Ablaufstrukturen der Wohlfahrtsverbände.

Entscheidend für die Strategie der örtlich agierenden Leistungsträger werden der lokale Markt und die dort vorfindbaren Wettbewerbsbedingungen sein. Chancen und Risiken müssen vor Ort - im Kontext der jeweiligen unterschiedlichen Rahmenbedingungen - verantwortet werden. Die konsequente Betonung der eigenen Alleinstellungsmerkmale, insbesondere die Beteiligungsmöglichkeiten der Nutzerinnen und Nutzer sowie die Fähigkeit zur Einbindung freiwilligen Engagements in die bestehenden Leistungsketten, können als Mittel dienen, die eigene Stellung unter gewandelten Rahmenbedingungen zu sichern und auszubauen.

Schon zum gegenwärtigen Zeitpunkt ist festzustellen, dass in den leistungsfähiger gewordenen Trägereinheiten vor Ort erhebliche fachliche und unternehmerische Kompetenzen entwickelt wurden, so dass sie in immer stärkerem Maße zu weitgehend autonomer Steuerung in der Lage sind und Leistungen des Spitzenverbandes kaum benötigen.

Die Kommunalisierungspolitik und die damit verbundene Wirkung auf die Träger und Einrichtungen, in lokalen Settings strategisch entscheiden und handeln $\mathrm{zu}$ müssen, werden die Zentrifugalkräfte in den Verbänden verstärken. Diese Entwicklung führt zur Abkopplung der lokalen Sozialbetriebe von den etablierten korporatistischen, lobbyistischen Strukturen ihrer Verbände. Der Aufbau eigener lobbyistischer Strukturen vor Ort wird dadurch zwingend notwendig. Mit Blick auf die Kommunalisierungsprozesse in Hessen schlussfolgert Manderscheid (2010) beispielsweise:

"Auf Ortsebene waren erstmals flächendeckend Liga-Strukturen notwendig. Mit der Kommunalisierung war das Land nicht mehr länger Ansprechpartner für die sozialen Dienste und Einrichtungen, die Kommunen wurden zum alleinigen Vertragsund Verhandlungspartner. Zugleich waren die Ortsligen nun gefordert, sich in die bereits bestehenden beziehungsweise die neu gegründeten Sozialplanungsgremien zu integrieren und dort ihre sozialpolitische Lobbyfunktion wahrzunehmen. Eine Umfrage der Liga bei den Ortsligen über die Erfahrungen mit der kommunalen Sozialplanung hat überwiegend positive Rückmeldungen ergeben: Auch wenn es einige, wenige Kommunen gibt, die nach wie vor die Wohlfahrtsverbände nicht beteiligen, hat sich doch die Zusammenarbeit zwischen Kommunen und Wohlfahrtsverbänden überwiegend verbessert. «

\section{Wohlfahrtsverbände: Anwalt der Betroffenen und sozialer Unternehmensverband}

Wohlfahrtsverbände bekommen oft die Frage gestellt, ob sie sich eher als Anwälte hilfesuchender Menschen begreifen oder als ein Unternehmensverband ihrer Mitgliedsorganisationen, die Träger von Diensten und Einrichtungen sind. Beide Funktionen gehören jedoch zusammen, da nur beide soziale Standards und soziale Angebote auf Dauern sichern können. [...]

Für die Ausgliederung von Unternehmungen aus Verbandsstrukturen kann es im Einzelfall eine Vielzahl guter Gründe geben. Die organisatorische Trennung kann in der Tat Probleme lösen und Verbände weiterbringen. Für das Problem von Interessenkollisionen in der Doppelfunktion von Spitzenverbänden - Unternehmensverbund und Sozialverband - stellt sie jedoch in aller Regel eine Scheinlösung dar.
Die Lösung besteht vielmehr in der steten und konstruktiven Bearbeitung des Spannungsverhältnisses selbst. Das heißt: Organisation von Kommunikation zwischen Unternehmungen und Verbandszentralen, offensives Aufgreifen der Problemanzeigen aus den Unternehmungen, eine Personalpolitik in den Verbandszentralen, die auch die Unternehmensbedürfnisse im Blick hat und ein mit Aufgeschlossenheit und Weitblick gesegnetes Management in den Unternehmungen selbst.

Dr. Ulrich Schneider, Hauptgeschäftsführer des Paritätischen Gesamtverbandes e. V., auf dem 5. Kongress der Sozialwirtschaft am 26. April 2007 in Magdeburg 
Die aktuelle Kommunalisierungspolitik führt zwar dazu, dass freie Träger sich schnell den neuen Gegebenheiten anpassen, eigene lokale Lobbying-Strukturen schaffen, dies aber nur selten als Herausforderung für eine konzeptionelle und fachliche Neupositionierung in der Sozialen Arbeit nutzen. Das Lobbying wird häufig der vorherrschenden betriebswirtschaftlichen Logik einverleibt und wird dadurch nicht selten dysfunktional: Betriebliche Umstrukturierungen ohne inhaltliche Orientierung verstärken die schon vorhandene betriebliche, wettbewerbsorientierte Logik der Träger mit allen Folgeproblemen. Die Kommunalisierungspolitik wird selten als Chance zur Schaffung neuer trägerübergreifender, klientenorientierter Vernetzungs- und Kooperationsstrukturen vor Ort genutzt. Man positioniert sich weiterhin als partikularisiertes Sozialunternehmen im Wettbewerb, muss aber aufgrund der durch die Kommunalisierungspolitik entstehenden Governance-Strukturen in einer Vielzahl von Verhandlungsarenen (Integration, Arbeitsmarkt, Jugendarbeitslosigkeit, Sicherheit u. Ä.) präsent sein, was die eigenen Transaktionskosten erhöht.

\section{Was Sozialunternehmen jetzt tun müssen}

Den Sozialunternehmen der Freien Wohlfahrtspflege wächst durch die Kommunalisierungspolitik eine neue gesellschaftspolitische Bedeutung zu, die sie bis- lang aber eher selten sehen und im Interesse ihrer Klienten nutzen. Während die Verbände die Sozialpolitik im alten Sozialstaat weitgehend dem Staat überlassen haben und sich auf fachpolitische Fragen bei der Implementation sozialpolitischer Aufgaben konzentriert haben, wird die Sozialpolitik, die sich zunehmend kommunalisiert, nun zu einem die Betriebe unmittelbar und im Eigeninteresse betreffenden Thema. Dabei geht es nicht nur um die Suche nach den jeweils geeigneten Instrumenten, um mit den jeweiligen Fortschritten sozialpolitischer Veränderungen Schritt zu halten, sondern um die Entwicklung eines Koordinatensystems, auf das sich das sozialpolitische Lobbying einigermaßen verlässlich beziehen kann.

Lobbying im Sinne der Anwaltsfunktion muss aber auch schon in die Struktur der Betriebe eingebaut werden. Das von der Sozialpolitik vorformulierte Förderprinzip "passgenauer « Unterstützungsleistung bringt - ernst genommen - eine klientenzentrierte Parteilichkeit in der Arbeit mit sich. Da die Arbeit mit Klienten, deren Bedarfe, zunehmend weniger von der Expertise der sozialen Professionen festgelegt, sondern vielmehr, extern, öffentlich-rechtlich definiert wird, muss dem Hilfeprozess selbst durch die Träger und Einrichtungen eine anwaltschaftliche Dimension eingebaut werden.

Verbands- und betriebsinterne Controllingsysteme, ein sozialraumbezogenes Monitoring und Instrumente der Armuts- und
Sozialberichterstattung müssen in Folge dieser Entwicklung schon in den sozialen Diensten implementiert werden, um auf Selektivitäten des Hilfesystems und Creaming-Effekte in einzelfallbezogenen Hilfen aufmerksam zu machen und um diese, wenn notwendig, auch öffentlich skandalisieren zu können.

Lobbying (im Sinne der alten Anwaltsfunktion) wird zu einem Bestandteil der Portfolio-Struktur der Sozialunternehmen und es wird eine verbandliche Aufgabe werden müssen, diese weiter zu entwickeln und mit Blick auf die Sicherung von Hilfeangeboten zu koordinieren.

\section{Anmerkungen}

(1) Das Konnexitätsprinzip formuliert den Grundsatz, dass Aufgabenwahrnehmung und Ausgabenverantwortung bei derselben staatlichen Ebene liegen sollen (Art. 104 a GG).

(2) "Zahlreiche Menschen in Not- und Konfliktsituationen suchen die Einrichtungen und Dienste der Caritas auf. Sie fragen um Rat oder Unterstützung nach. Indem sie die Mitarbeiterinnen und Mitarbeiter der Caritas konsultieren, erteilen sie im klassischen Sinne von Anwaltschaft einen Auftrag. Sie wünschen, dass wir uns für sie oder mit ihnen in einer Sache engagieren und gemeinsam nach Lösungen suchen." (Puschmann 1999, S. 182)

\section{Neu im Studienkurs}

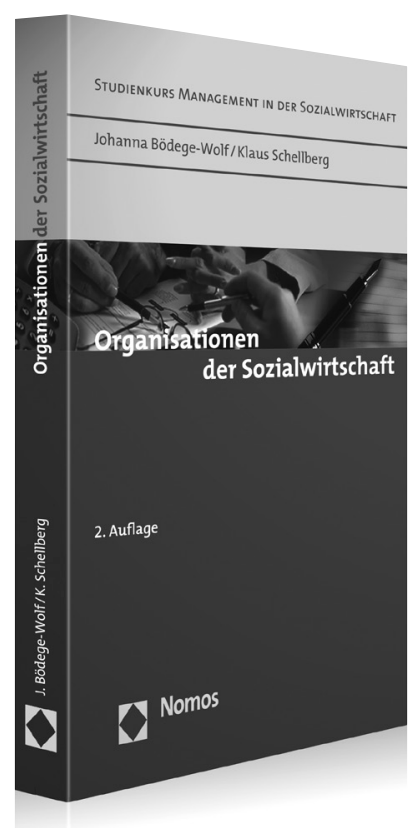

\section{Organisationen der Sozialwirtschaft}

Von Prof. Dr. Johanna Bödege-Wolf und Prof. Dr. Klaus Schellberg

2. Auflage 2010, ca. 180 S., brosch., ca. 19,80 €, ISBN 978-3-8329-5206-8

(Studienkurs Management in der Sozialwirtschaft)

Erscheint ca. Juli 2010

Der Band ist für ein fundiertes Verständnis der verschiedenen Organisationen des Sozialsektors und ihrer Managementaufgaben unerlässlich. Die Autoren liefern einen aktuellen Überblick über Strukturen, Rechtsformen, ideelle Grundlagen, Entwicklungslinien, Formen der Zusammenarbeit und Managementaufgaben der freien und öffentlichen Träger sowie der Dienstleistungsunternehmen.

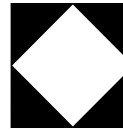

